\title{
Correlation between Radial Velocities with Diameter of Abdominal Aortic Aneurysms from MRI
}

\author{
C.M. Karyati ${ }^{\# 1}$, A. Muslim ${ }^{\# 2}$, S. Widiyanto ${ }^{* 3}$, Febriani ${ }^{\# 4}$, A.B. Mutiara ${ }^{\# 5}$ \\ ${ }^{\#}$ Faculty of Computer Science and Information Technology, Gunadarma University \\ Jl.Margonda Raya 100, Depok 16424, Indonesia \\ * Faculty of Industrial Technology, Gunadarma University \\ Jl.Margonda Raya 100, Depok 16424, Indonesia \\ e-mail: csyarah, amuslim, swidiyanto, febriani, amutiara@staff.gunadarma.ac.id
}

\begin{abstract}
The main parameters to determine the medical treatment for AAA is the size of diameter. Several parameters have been proposed to predict the evolution of the aorta diameter, such as thrombus existence, blood flow velocity, flow rate and shear stress on aortic wall. This article proposes profile of blood flow velocity that moving toward aortic wall (Radial Velocity) that causing presumption that will push up the size of the aortic wall. Data were obtained from 11 patients with Small Abdominal Aortic Aneurysms (SAAA) who had an examination at least 1 to 3 times with an examination every 6 to 12 months (according to the patient). MR Images were acquired on a $3 T$ Imager. Based on the proposed formula to obtain the value of the radial velocity, it can be conclude that the radial velocity value is have the good correlation to the value of the maximum diameter with $R=0,736$ (where $0,5>R>1$ ).
\end{abstract}

Keywords: Evolution of Diameter, Radial Velocity, Abdominal Aortic Aneurysm

\section{INTRODUCTION}

Until now, the main parameter to determine the appropriate action for AAA is the size of diameter. Several parameters has been proposed to predict the evolution of aortic wall diameter, for instance are thrombus existance, blood flow velocity, volume of blood flow, flow rate, and shear stress. The observation using MRI is still considered to be very supportive of image processing to obtain the relationship between those paramters with the evaolution of AAA diameter.

Previous research has suggested thrombus as parameter that supports evolution of aortic diamter [Karyati, 2011]. In those studies have not obtained high correlation between categories of founded thrombus with the measurement of diameter evolution.

The further researches get 3D blood flow velocity value and represent 2D and 3D animation of blood flow on AAA with or without thrombus. 3D blood flow velocity value has been obtained and also good correlation with aortic diameter [Karyati, 2011].

The paramters of blood flow volume at AAA have also been obtained in previous research by reconstructing the blood flow image from MRI observation result. In this case, the volume of blood flow is not directly proportional to the evolution of aortic diamter [Karyati, 2014].

As an advance of previous research by author, the curent research will present one profile of blood flow velocity thar moves to touch the walls of aorta. Blood flow velocity profile is called radial velocity. Radial velocity is expected that will be have positive correlation with evolution of aortic diameter value, then this value can be used to get the value of shear stress that allegedly could be a parameter to predict rupture of the aortic wall.

\section{RESEARCH METHODS}

\section{A. Blood Flow Velocity}

Blood flow velocity in humans normally range between $20-22 \mathrm{~cm} / \mathrm{sec}$ at abdominal aorta size in between $1 \mathrm{~cm}-2 \mathrm{~cm}$ [Samad, 2012]. From the measurement of blood flow velocity in non-hypertension patients obtained results ranged from $20.9 \mathrm{~cm} / \mathrm{sec}$ to $22.9 \mathrm{~cm} / \mathrm{sec}$ with the highest blood pressure was $130 / 90 \mathrm{~mm} / \mathrm{Hg}$ which produces blood flow velocity $22.7 \mathrm{~cm} / \mathrm{sec}$, while in hypertensive patients blood velocity values ranging from $13.5 \mathrm{~cm} / \mathrm{sec}$ to $19.5 \mathrm{~cm} / \mathrm{sec}$ with the highest blood pressure at $200 / 100 \mathrm{mmHg}$, and produce blood flow velocity 
in $13.5 \mathrm{~cm} / \mathrm{sec}$. According to the theory of Fluid Mechanics, large blood pressure is directly proportional to the blood flow veloccity. In hypertension cases are usually accompanied by a decrease of blood vessel walls elasticity which increases the resistance, and this resistance will decrease the blood flow velocity. In Figure 1 can be seen smooth blood flow (normal) in a normal aorta.

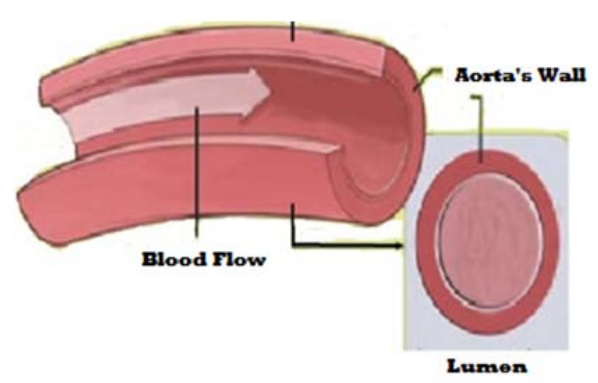

Fig.1. The normal blood flow

[Office for Technology Commercialization-University of Minnesota, 2010]

Radial velocity method (also known as Doppler Spectroscopy or Doppler Method) has long been applied to calculate radial rotation speed of 300 extra solar planets discovered to date. A lot of paper have been derived the theoretical equation that was associated with the variation over time of a star's velocity along an observer's line-of-sight [Clubb, 2014]

A quantity can be precisely measured with an optical telescope. Begin with the simple situation of a planet orbiting a star and then applying basic principles of physics that utilize mathematical tools in order to analyse and describe the star planet system and found a complete and detailed derivation of the Radial Velocity. Equation illustrated by Figure 2-3.

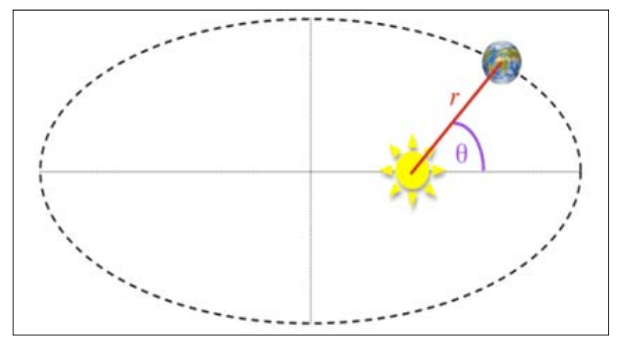

Fig. 2. As the planet orbits its host star, the distance between the star and planet changes [Clubb, 2014]

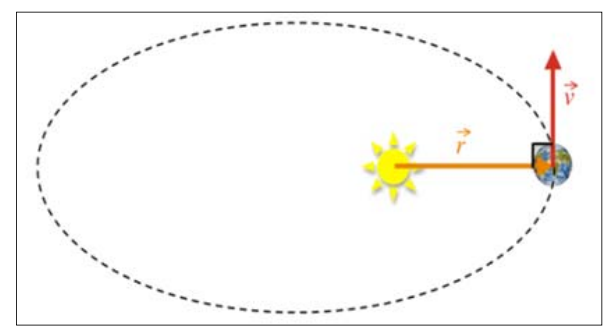

Fig. 3. As the planet orbits its host star, its position vector $\vec{r}$ and $\vec{v}$ are always perpendicular [Clubb, 2014]

\section{B. Patients}

Blood flow velocity in humans normally range between $20-22 \mathrm{~cm} / \mathrm{sec}$ at abdominal aorta size in between 1 cm - 2 Data were obtained with MRI 3T from 11 patients with Small Abdominal Aortic Aneurysms Study (SAAA) who had been examined for 2-3 years. Each patient has had an examination at least 1 to 3 times with an examination every 6 to 12 months (according to the patient). 
TABLE I

Characteristic of Patients

\begin{tabular}{|l|l|l|l|}
\hline Name of Patient & Sex & $\begin{array}{l}\text { Age } \\
\text { (year) }\end{array}$ & Characteristic \\
\hline Patient 1 & Female & 68 & Dyslipidemie \\
\hline Patient 2 & Male & 62 & Smooking, Hypertensi, Dyslipidemie \\
\hline Patient 3 & Male & 59 & Ex Smooking \\
\hline Patient 4 & Male & 79 & Ex Smooking, Hypertensi, Dyslipidemie \\
\hline Patient 5 & Male & 77 & Ex Smooking, Hypertensi, Dyslipidemie \\
\hline Patient 6 & Male & 71 & Smooking, Dyslipidemie \\
\hline Patient 7 & Female & 74 & Ex Smooking \\
\hline Patient 8 & Male & 55 & Ex Smooking, Hypertensi, Dyslipidemie \\
\hline Patient 9 & Male & 51 & Ex Smooking, Dyslipidemie \\
\hline Patient 10 & Male & 73 & Ex Smooking, Hypertensi, Dyslipidemie \\
\hline Patient 11 & Male & 59 & Smooking \\
\hline
\end{tabular}

\section{Methods}

The All patients had been processed on Thrombus analysis with T1 and T2 weighted image to calculate maximum diameter of the aorta [Karyati, 2011], and had been processed too on Blood Flow analysis with Magnitude/Amplitude image and Phase Contrast Image to calculate velocity profile. DICOM Work has been used to import data from MRI examination and to export images to support image processing with MatLab Software. For each patient, these images were located at the same position in one examination to another. Processing was done in T1 and T2 weighted images in order to determine diameter aorta. The diameters are at three positions, such as: Anterior-Posterior Diameter, Transversal Diameter and maximum diameter. Maximum aortic diameter was automatically obtained from automatic tracing on $\mathrm{T} 1$ weighted images in all examinations. After then we calculate the evaluation of the aortic diameter [Karyati, 2011].

After using the T1 and T2 weighted images to determine aortic diameter, we use Magnitude Images and Amplitude Images from MRI examination to analyze blood flow in the aorta. The acquisition is taken perpendicular at the level of abdominal aorta and done while free breathing or breathe hold in accordance with the localization [Haacke, 1999]. By using those images, we can obtain the value of blood flow velocity on the axis $\mathrm{x}, \mathrm{y}$ and $\mathrm{z}$ of three direction, such as the position from right to left (right-left), from front to back (anteriorposterior), and from top to down (through-plane) in one examination to another examination [Hollenberg, 2006].

Processing was done in all examination in order to calculate the value of speed of the blood flow on SAAA. Blood fow velocity $(\mathrm{cm} / \mathrm{s})$ is a value equal to the total volume flow divided by the cross-sectional area of the vascular. The maximum 3D blood flow velocity has been calculates on the previous paper [Karyati, 2011].

To assess the hemodynamic stress on the aortic wall must be determined beforehand radial component of the velocity vector which is closest to the aortic wall. Light gravity center placement of the aorta is very important. The gravity center is a point that has coordinates G (average X, average Y). Furthermore, the point of cloud/shadow/cloud itself taken from the point of pixels on the edge of the aortic wall as illustrated in Figure 4.

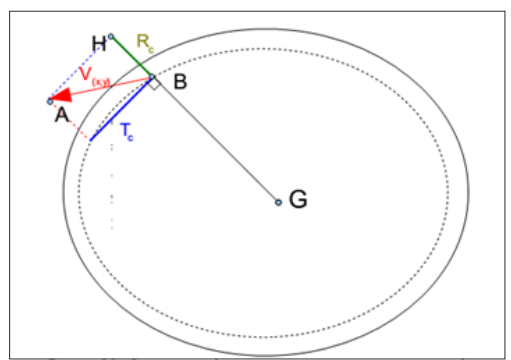

Fig.4. Illustration of radius on searching radial velocity values [Xavier, 2007]

BA defines speed from "right/left" and "front/rear." Velocity vector has a radial R component and ortho-radial component. Based on the coordinates of the center of gravity "G" and pixel coordinates "B" that closest to the aortic wall, it can be obtained equation of the line BG. Thus it can be also obtained several formulas to calculate values based on the radial velocity vector ortho-radial which can be seen in eq. 1-3. [Xavier, 2007]

$$
\begin{aligned}
& A H=\frac{\left|a x_{A}-y_{A}+b\right|}{\sqrt{a^{2}+1}} \\
& B H=\sqrt{A B^{2}-A H^{2}}=\sqrt{\left(\left(x_{B}-x_{A}\right)^{2}+\left(y_{B}-y_{A}\right)^{2}\right)-A H^{2}}
\end{aligned}
$$


So that,

$$
\text { Max Radial Velocity }(t)=\sqrt{V^{2} x \max +V^{2} y \max -V^{2} 3 D \max }\left(\frac{\mathrm{cm}}{\mathrm{s}}\right)(3)
$$

To represent the radial blood flow, the coordinates of the point $\mathrm{H}$, which is orthogonal projections of the velocity vector at the end of the line through the initial vector velocity and centre of gravity, must be founded.

\section{RESULTS AND ANALYSIS}

\section{A. Results}

Blood Earned value of radial velocity of blood flow compared with the evolution of aortic diameter is shown in Table 2.

TABLE 2

Results

\begin{tabular}{|c|c|c|c|c|}
\hline Patient & $\begin{array}{c}\text { Thrombus } \\
\text { Categories }\end{array}$ & $\begin{array}{c}\text { Diameter } \\
\text { Maximum } \mathbf{( c m )}\end{array}$ & $\begin{array}{c}\text { Evolution of } \\
\text { Diameter (cm/year) }\end{array}$ & $\begin{array}{c}\text { Radial Velocity } \\
\mathbf{( c m} / \mathbf{s})\end{array}$ \\
\hline P1 & No thrombus & 5,7 & 0.061 & 6.125 \\
\hline P2 & No thrombus & 3,5 & 0,01 & 10,64 \\
\hline P3 & With thrombus & 5,7 & 0,033 & 4,89 \\
\hline P4 & With thrombus & 3 & 0,01 & 7,17 \\
\hline P5 & With thrombus & 4,8 & 0 & 6,38 \\
\hline P6 & With thrombus & 4,7 & 0 & 3,92 \\
\hline P7 & With thrombus & 5,7 & 0,012 & 7,6 \\
\hline P8 & With thrombus & 5 & 0,011 & 6,09 \\
\hline P9 & With thrombus & 4,4 & 0 & 13,14 \\
\hline P10 & With thrombus & 4,1 & 0 & 13,37 \\
\hline P11 & With thrombus & 3,5 & 0 & 22,22 \\
\hline
\end{tabular}

In Figure 5a showed blood flow in 4D format, the in Figure 5b represent 2D image to show the direction of radial velocity in AAA. Flow evolution during the cardiac cycle represented in Figure 5c.

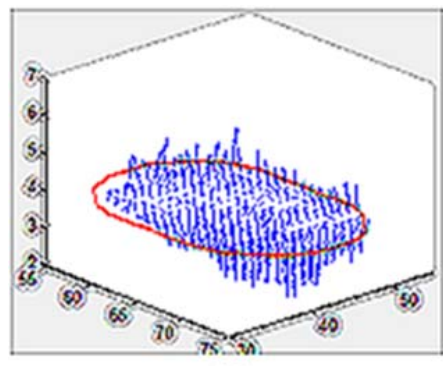

(a)

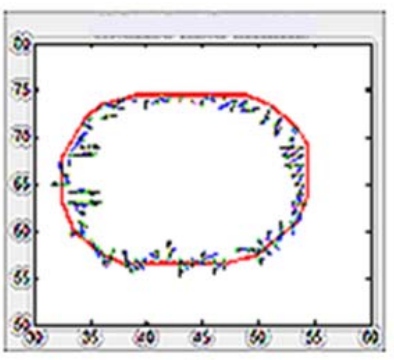

(b)

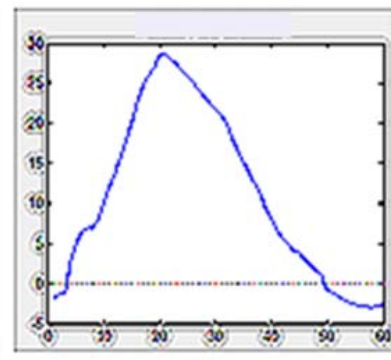

(c)

Fig. 5. Diameter maximum $=4,4 \mathrm{~cm}$, with thrombus, Acquisition speed : $100 \mathrm{~cm} / \mathrm{s}(\mathrm{DG}), 100 \mathrm{~cm} / \mathrm{s}$ (AP), $200 \mathrm{~cm} / \mathrm{s}$ (TP). a) blood flow in $4 \mathrm{D}$ format, b) radial velocity in $2 \mathrm{D}$ format, c) blood flow evolution during cardiac cycle

\section{B. Analysis}

It has been obtained good correlation for the comparison value in the radial velocity with a maximum diameter of the aorta. These results indicate that between radial velocity in each category of thrombus signal with a maximum diameter of the aorta there is a linear relationship $(\mathrm{y}=1,3257 \mathrm{x}+0,7202)$, where this is indicated by the discovery of the value of $r=0,736(0.5<\mathrm{r}<1)$ in patients SAAA. It can be seen in the following graph: 


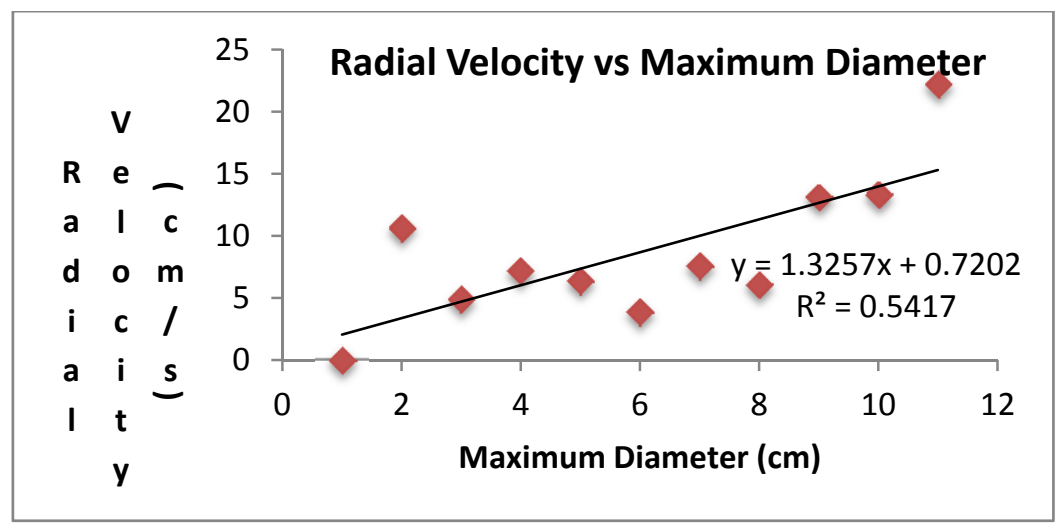

Fig. 6. Correlation between Radial Velocities with Maximum Diameter

But, there are no good correlation between radial velocity and evolution of aortic diameter on SAAA where the values of $r=0,439(r<0,5)$ as can be seen at figure 7 .

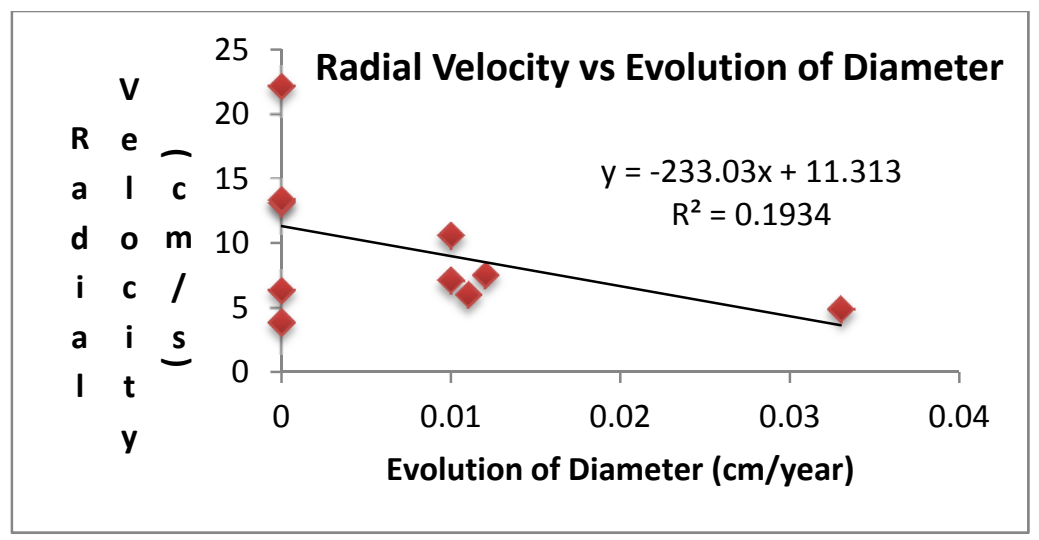

Fig. 7. Correlation between Radial Velocities with the Evolution of Diameter

The value of the evolution of diameter is very small where less than $0.5 \mathrm{~cm}$ per year. It has become an obstacle in finding a significant correlation between the values of radial velocity with the value of the evolution of diameter. It is also because the data used is the observation of patients with small aneurysm (diameter $<5.5$ $\mathrm{cm}$ ) with the condition there are some patients who did not have an evolution of diameter at each examination (the evolution of diameter $=0 \mathrm{~cm} /$ year).

\section{CONCLUSION}

Based on the comparative analysis that has been done by linear regression method, it can be concluded that the value of the radial velocity has a linear correlation with the value of the maximum diameter, or in other words, the greater the value of the maximum diameter, then the value of the radial velocity will also be higher.

But, the same correlation does not happen on the relationship between the value of radial velocity with the value of evolution in diameter. So we can say that on the SAAA, where the value of the evolution of the diameter per year is less than $0.5 \mathrm{~cm}$ will not affect the value of the radial velocity and vice versa.

\section{REFERENCES}

[1] Clubb, 2014, A Detail Derivation of the Radial Velocity Equation, San Francisco State University, www.physics.sfsu.edu

[2] Haacke, 1999, MRI: Physical Principles and Sequence Design, Canada

[3] Hollenberg, 2006, Modeling, Analysis and Visualisation, Lecture 21, pp. 433-385

[4] Karyati C.M., Lalande A., Steinmetz E., Mutiara A.B., Brunotte F., 2011, Prediction of the Evolution of the Aortic Diameter According to the Trombus Signal from MR Images on Small Abdominal Aortic Aneurysms, Medical and Health Science Journal (MHSJ), Volume 5, Pages 49-56

[5] Karyati C.M., Refianti R., Muslim A., Mutiara A.B., 2011, Effect of Thrombi on Blood Flow Velocity in Small Abdominal Aortic Aneurysms from MRI Examination, International Journal of Advanced Computer Science and Applications (IJACSA), Volume 2, No.3

[6] Karyati C.M., Widiyanto S., Madenda S., Harlan J., Mutiara A.B., 2014, Reconstruction 4D of Blood Flow MR Imaging on Abdominal Aortic Aneurysms with Thrombus Signal, Journal of Theoretical and Applied Information Technology (JATIT), Volume 63, No. 1, pages 119-124

[7] Office for Technology Commercialization-University of Minnesota, 2010, Percutaneous Transluminal Devices and Angioplasty and Atherectomy Risks, available on www.license.umn.edu

[8] Samad S., 2012, Analysis of Blood Flow Velocity on Abdominal Aorta for Patients with Hypertension using Color Doppler Sonography, Medical Physics, University of Hasanuddin

[9] Xavier M., Lalande A., Walker P.M., Boichot C., Cochet A., Bouchot O., Steinmets E., Legrand L., Brunotte F., 2007, Dynamic 4D Blood Flow Representation in the Aorta and Analysis from Cine-MRI in Patients, Computers in Cardiology, 34:375-378 